\title{
Hydrocephalus induced via intraventricular kaolin injection in adult rats
}

\author{
Zhang Shaolin ${ }^{1,2}$, Wang Zhanxiang ${ }^{1}$, Xu Hao ${ }^{1}$, Zhang Feifei ${ }^{1}$, Huang Caiquan ${ }^{1}$, Chen Donghan ${ }^{1}$, Bao Jianfeng ${ }^{3}$, \\ Liu Feng ${ }^{1}$, Shen Shanghang ${ }^{1}$ \\ ${ }^{1}$ Department of Neurosurgery, The First Affiliated Hospital of Xiamen University, Xiamen 361003, China, ${ }^{2}$ Department of Neurosurgery, \\ The First Hospital of Nanping, Nanping, Fujian 353000, China, ${ }^{3}$ Fujian Provincial Key Laboratory of Plasma and Magnetic Resonance, \\ State Key Laboratory of Physical Chemistry of Solid Surfaces, Xiamen University, Xiamen 361003, China
}

\begin{abstract}
Hydrocephalus is a common neurological disease in humans, but a uniform and particularly effective hydrocephalic animal model amenable to proper appraisal and deep study has not yet been established. In this study, we attempted to construct a high-efficiency model of hydrocephalus via intraventricular kaolin injection. Adult male SpragueDawley rats were randomly divided into 2 groups: the control group $(n=15)$ and the experimental group $(n=30)$. Kaolin was injected into the lateral ventricle of experimental animals. Control rats underwent the same procedure but received sterile saline injection instead of kaolin. All animals with kaolin injection into the lateral ventricle developed hydrocephalus according to magnetic resonance imaging (MRI) results (success rate up to 100\%). Also, the Morris water maze (MWM) test demonstrated disturbed spatial learning and memory. Furthermore, there were significant differences between groups with respect to the histological changes in the periventricular tissue. Our results indicate that experimental hydrocephalus induced by lateral ventricle injection of kaolin in adult rats is feasible and may be widely used.
\end{abstract}

Key words: kaolin, hydrocephalus, animal model, MRI, Morris water maze.

\section{Introduction}

Hydrocephalus is a common neurosurgical disease that is often the result of intracranial hemorrhage, tumor, intracranial infection and brain injury. Abnormalities of cerebrospinal fluid (CSF) secretion, circulation and absorption cause excessive accumulation of CSF in the ventricular system and expansion of the ventricular system, which may cause damage of surrounding brain tissue and persistent neurological deficits, leading to the development of hydrocephalus.

The sustained enlargement of cerebral ventricles can result in compression and distortion of brain tissue. Compression and distortion of brain tissue give rise to deleterious effects, such as inflammatory responses, gliosis, fiber stretching, damage to neurons and cellular pathways, destruction of periventricular axons, demyelination, reduced cerebral blood flow and oxygen levels, and altered clearance 
of proteins and toxins [8-10,22,25,33], that may be associated with neurological deficits in hydrocephalus patients, including low intelligent quotient (IQ) scores, learning disabilities, memory loss, mental retardation, impaired gait, and urinary incontinence $[12,13]$. These deleterious effects of hydrocephalus on the brain depend on the magnitude and duration of ventriculomegaly and degree of compression of brain tissue, which are also modified by the age of onset [9].

Many animal models of hydrocephalus have been established, but most models mimic "obstructive" hydrocephalus by blocking CSF flow at the $4^{\text {th }}$ ventricle outlets $[21,26,27,29,34]$. Communicating hydrocephalus has been difficult to model in rodents because the subarachnoid spaces are extremely small and difficult to access. Transgenic models [15], growth factors such as TGF- $\beta 1$ and FGF-2 [24,30], neurotoxins [14], and viral [6] and bacterial inoculations [32] have also been used to produce communicating hydrocephalus. However, these methods have not been widely applied because of complexity, costliness, low success rate and high acute mortality, etc.

Although hydrocephalus can be experimentally induced through a variety of techniques $[1,15,29,30]$, the most widely used method of experimental hydrocephalus involves induction by injection of kaolin into the cistern magna in neonatal, juvenile and adult rodents [16,21]. Ventricular enlargement occurs as a result of the inflammatory scarring, which causes an obstruction of the CSF pathways near the 4th ventricle outlets $[16,21,23]$. This method is long-term and widely used; however, there are also many deficiencies (such as high acute mortality rates, low success rate, difficulty to form a communicating hydrocephalus and uncontrollable hydrocephalic severity) $[2,4,21,29]$.

It is well known that communicating hydrocephalus, clinically, is more universal than obstructive hydrocephalus. For a long time, the lack of appropriate animal models of communicating hydrocephalus has clearly restricted investigative progress of this disease. Recently, communicating hydrocephalus with suspension of $25 \%$ kaolin injections into the basal cisterns or the cortical subarachnoid space of adult rats has been reported $[18,23]$. So, theoretically, if low concentration kaolin injections into the lateral ventricle may induce hydrocephalus, it should be communicating hydrocephalus.
Thus, we attempted to explore and develop a uniform, particularly effective and low mortality animal model of hydrocephalus.

\section{Material and methods}

\section{Animals}

Adult male Sprague-Dawley rats (240-260 g) were purchased from the Slac Laboratory Animal Co. Ltd. (Shanghai, China). The animals were kept in a temperature- and humidity-controlled room, allowed food and water freely, and housed in the same animal care facility during a 12-h light/dark cycle throughout the protocol. The rats were randomly divided into 2 groups: the experimental group $(n=30)$ with kaolin injection; and the control group ( $n=15)$ with saline injection. All animal care and experimental procedures were licensed and carried out by the Local Institutional Animal Care and Use Committee according to the Institutional Animal Care and Use Committee guidelines.

\section{Surgical induction}

Rats were anesthetized by intraperitoneal injection of $10 \%$ chloral hydrate $(0.4 \mathrm{ml} / 100 \mathrm{~g})$, and were placed in a stereotaxic frame. The scalp was sterilized and incised, and the bregma was exposed. The injection coordinates, which were measured from the bregma to the lateral cerebral ventricles, were $0.8 \mathrm{~mm}$ posterior, $1.6 \mathrm{~mm}$ lateral and $3.7 \mathrm{~mm}$ deep. A $50 \mu \mathrm{l}$ syringe with 30-gauge needle was inserted into the lateral ventricle (depth: $3.7 \mathrm{~mm}$ ), and a $30 \mu \mathrm{l}$ sterile suspension of 3\% kaolin (ultrasonic emulsification about 15 minutes) in saline was injected slowly into the lateral ventricle at a rate of approximately $10 \mu \mathrm{l} / \mathrm{min}$. The skin incision was sutured, and the animal was replaced to its cage and was monitored daily for the duration of the experiment. Control group rats underwent the same procedure but received sterile saline injection instead of kaolin.

\section{Morris water maze test}

Ten days later, spatial learning and memory was examined with the Morris water maze (MWM) test. The water maze consisted of a black pool $(1.5 \mathrm{~m}$ in diameter, $50 \mathrm{~cm}$ high, bottom $45 \mathrm{~cm}$ above floor level) filled with water (made opaque with ink) and a black platform (10 $\mathrm{cm}$ in diameter, $30 \mathrm{~cm}$ high) 
submerged $2 \mathrm{~cm}$ below the water surface. The quadrant in which the platform was placed and the remaining quadrants, clockwise from the former, were designated " 1 ", " 2 ", " 3 " and " 4 ", respectively. The water was maintained at $21 \pm 1^{\circ} \mathrm{C}$, and the platform was placed in the " 1 " quadrant of the four virtual quadrants $30 \mathrm{~cm}$ away from the sidewall. The movements of rats were recorded with a video camera connected to a computer. Data were analyzed using a tracking program (DigBehv-MWM, Shanghai Jiliang Software Technology Co. Ltd., Shanghai, China). The experimental room $(2.4 \times 2.4$ $\times 3.2 \mathrm{~m}$ ) contained cues: a pole, maze, door, computer, air conditioning, and researcher. A quiet and constant environment (including temperature and humidity) of the laboratory was maintained during the experiment.

For maze performance, a 5-day protocol was used (day 1: visual cue trial; days 2-4: 3 trials per day, hidden platform; day 5: probe trial with platform removed). On the cue and training days, each rat was placed at one of the other three starting points, which were used in a random order so that each position could be used once. A $1 \mathrm{~h}$ interval was imposed before the beginning of the next trial. If the rat did not find the hidden platform within 60 seconds, it would be guided to find the platform and remain for 15 seconds. The platform location was immovable during the cue and learning period. The time that rats spent in finding the submerged platform (escape latency) was recorded in each trial. On the probe day, the platform was removed from the pool and each rat was given one 60-second trial in which the starting point was quadrant 3 located in the most distant position to the former platform. The number of times of crossing the former platform position and the swimming time in each quadrant of the pool without the platform were recorded and analyzed as the score. All the experiments were performed at 08:00 AM.

\section{Magnetic resonance imaging and assessment of ventricular size}

All MRI experiments were performed on a Varian 7.0 T small animal scanner with a 63/95-mm-quad birdcage coil. The imaging parameters were as follows: T2-weighted spin-echo imaging; three orientations (coronal, axial, sagittal); section thickness: $1 \mathrm{~mm}$; number of slices were enough to cover the ventricle system area; TR: $5000 \mathrm{~ms}$; TE: $50 \mathrm{~ms}$; fieldof-view: $5.0 \times 5.0 \mathrm{~cm}$; matrix size: $128 \times 128$; number of signals acquired: 1 . The total imaging time was $17 \mathrm{~min}$. Magnetic resonance imaging (MRI) was performed on all animals 15 days after the injection (after the MWM test).

The maximal width of the ventricles at the level of the foramen of Monro was taken as the cerebral ventricle diameter because these images had the widest ventricular space and were common in these MRI series. Ventricular volumes were measured from the coronal MRI scans of the saline control and hydrocephalic brains. The volumetric calculations of the ventricle were semiautomated by image analysis software (NIH Image) as follows: an appropriate intensity threshold was first chosen to exclude background tissue and to highlight the bright ventricles. This was followed by careful inspection of each image, and manual tracing was used to correct any areas of the ventricle that had been incorrectly deleted, or to delete non-ventricular regions that had been incorrectly included. This process resulted in a binary mask of ventricular pixels, which when multiplied by the volume of each pixel and summed over all slices produced the net ventricular volume in milliliters.

\section{Sacrifice and histological analyses}

On post-injection day 16 following $M R I$, rats were anesthetized with $10 \%$ chloral hydrate $(0.4 \mathrm{ml} / 100 \mathrm{~g}$ i.p.) and perfused with $0.9 \%$ saline by left atrial perfusion. For histological analysis, the brains were removed from the skull, fixed in 10\% formalin for $24 \mathrm{~h}$ at $4^{\circ} \mathrm{C}$ and then embedded in paraffin. The brain was sliced coronally into a thickness of $5 \mu \mathrm{m}$ at the level of the lateral ventricle and then stained with hematoxylin and eosin (H\&E).

\section{Statistical analysis}

All data were analyzed using GraphPad Prism (GraphPad, Inc., La Jolla, CA, USA) software to compare differences between groups. The data from the MWM were expressed as mean \pm SEM and the ventricular volume was expressed as the median value (range). Statistical analysis of these MWM data was performed by two-way analysis of variance (ANOVA), and the ventricular volume was assessed by the Mann-Whitney $U$ test. Statistical values of $p<0.05$ were considered to be significant. 


\section{Results}

\section{Mortality, success rate, behavior and general changes}

Rats were monitored and weighed daily. Two rats (hydrocephalic animals: 1 rat; controls: 1 rat) died from anesthesia or surgical trauma within 2 days after the operation. Forty-three rats (hydrocephalic animals: 29 rats; controls: 14 rats) participated in the entire course of the experiment. In the experimental group, 29 rats successfully developed moderate to severe hydrocephalus according to MRI examination (success rate up to $100 \%$ ). Most rats of the hydrocephalus group exhibited coughing, irritability, emaciation, unsteady gait, increased urine, hunched back, hind legs weak and nasal and/or orbital secretions of blood and clear fluid after 1 post-operative day. These signs disappeared spontaneously within a few days. Weight growth of hydrocephalic animals was significantly slower than that of control animals.

\section{Morris water maze test}

Figure 1 shows the results of the MWM test. The hydrocephalus group tended to require more time to find the platform than the control group, and there was a significant difference between the two groups on the $3^{\text {rd }}$ day and the $4^{\text {th }}$ day $(p<0.05)$ (Fig. $\left.1 \mathrm{~A}\right)$. The control group spent a greater proportion of their swimming times in quadrant 1 that contained the hidden platform $(27.86 \pm 3.195 \mathrm{~s})$, and there was a significant difference compared with the others ( $p<0.05)$. In contrast, the mean swimming time of the hydrocephalus group in quadrant 1 was only $20.26 \pm 3.166 \mathrm{~s}$, and there was only a significant difference compared with quadrant 2 (Fig. 1B). The number of times of crossing the former platform position of hydrocephalic rats was higher than that of controls, but statistical significance was not reached (data not shown). All results reflected a decline of spatial learning and memory in hydrocephalic rats.
A

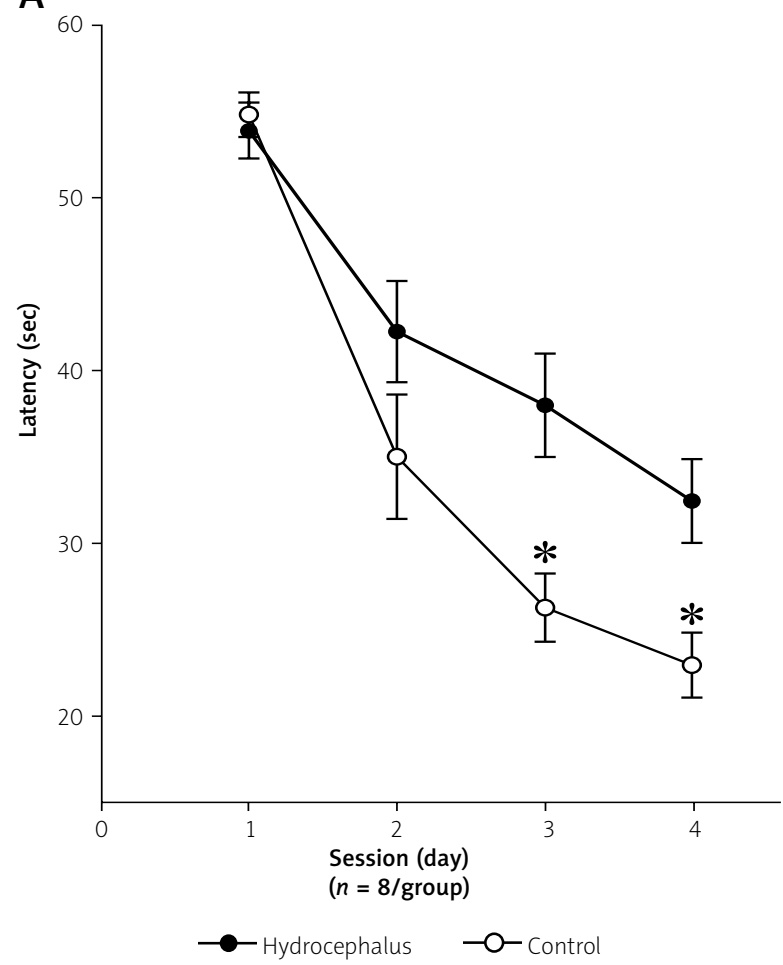

B

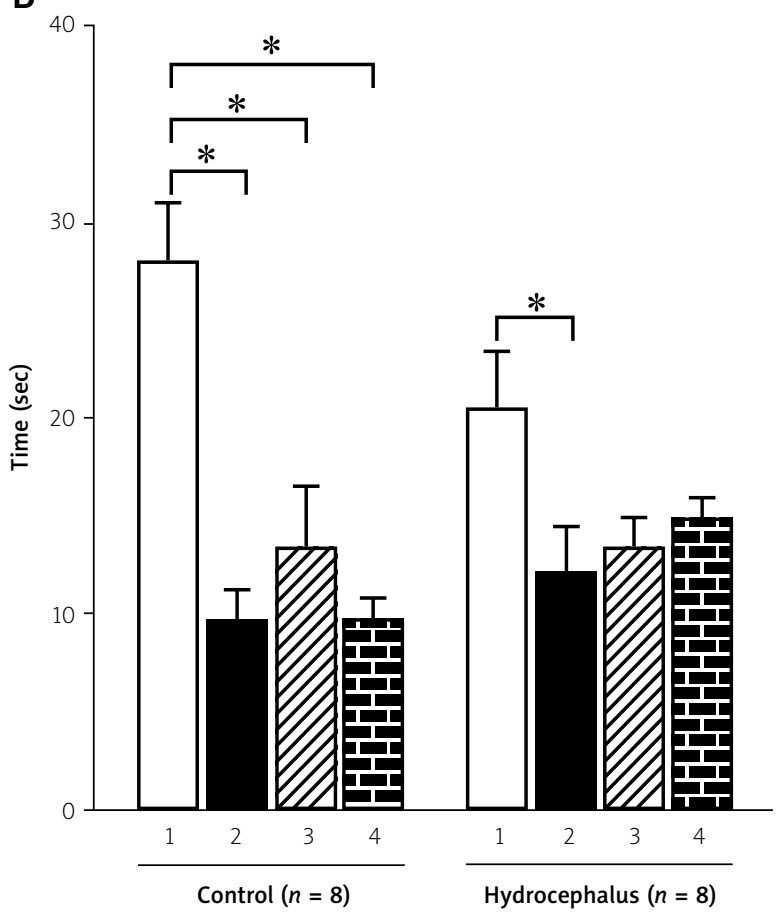

Fig. 1. A) Graph showing the mean escape latencies for finding the hidden platform. The mean escape latency in hydrocephalic rats was markedly longer compared with control rats, and there was a significant difference between the two groups on the $3^{\text {rd }}$ and $4^{\text {th }}$ day. B) Graph showing the swimming time in each quadrant of the pool without the platform. 1 is the quadrant in which the platform was initially placed, and 2,3 and 4 are the remaining quadrants. The control rats spent a greater proportion of their swimming times in quadrant $1(27.86 \pm 3.195 \mathrm{~s})$ than the hydrocephalus group $(20.26 \pm 3.166 \mathrm{~s}) .{ }^{*} p<0.05$. 


\section{Magnetic resonance imaging and the enlarged ventricular system}

Animals with saline injection into the lateral ventricle did not develop hydrocephalus; however, all rats with kaolin injection developed hydrocephalus. In these animals, MRI confirmed ventriculomegaly involving all ventricles (including the cerebral aqueduct, the third ventricle and the frontal horns and temporal horns of the lateral ventricle, etc.), and compression and thinning of the cortical tissue (Fig. 2A). Row 1 shows control rats at 16 days after saline injection. Row 2 shows hydrocephalic rats (16 days after kaolin injection). In comparison to the controls, there was obvious enlargement of all portions of the cerebral ventricles. General specimens show that control rats presented the normal configuration of the ventricular system, but experimental animals displayed significant expansion in the anterior horns and temporal horns of the lateral ventricle, midbrain aqueduct and fourth ventricle, and compression, thin- ning, fracture and distortion of the callosum and cortical tissue after kaolin injection (Fig. 2B). The lateral ventricles and the cerebral aqueduct were enlarged at the different sections and positions, and the fourth ventricle outlets remained open. Therefore, it corresponded to the classification of communicating hydrocephalus. The ventricular volume was detected in all the control rats (median value: $15.65 \mathrm{~mm}^{3}$; range: $8.78-31.52 \mathrm{~mm}^{3} ; n=14$ ), but in all the hydrocephalic rats (median value: $147.95 \mathrm{~mm}^{3}$; range: 58.51 $222.27 \mathrm{~mm}^{3} ; n=29$ ). The ventricles were significantly dilated in the hydrocephalic rats compared to the controls $(p<0.05)$ (Fig. 3).

\section{Histopathological findings}

There was a significant difference between groups with respect to the histopathological changes in the vicinity of the lateral ventricle. Compared with the controls, the hydrocephalic animals showed advanced periventricular reactive astrogliosis with

A
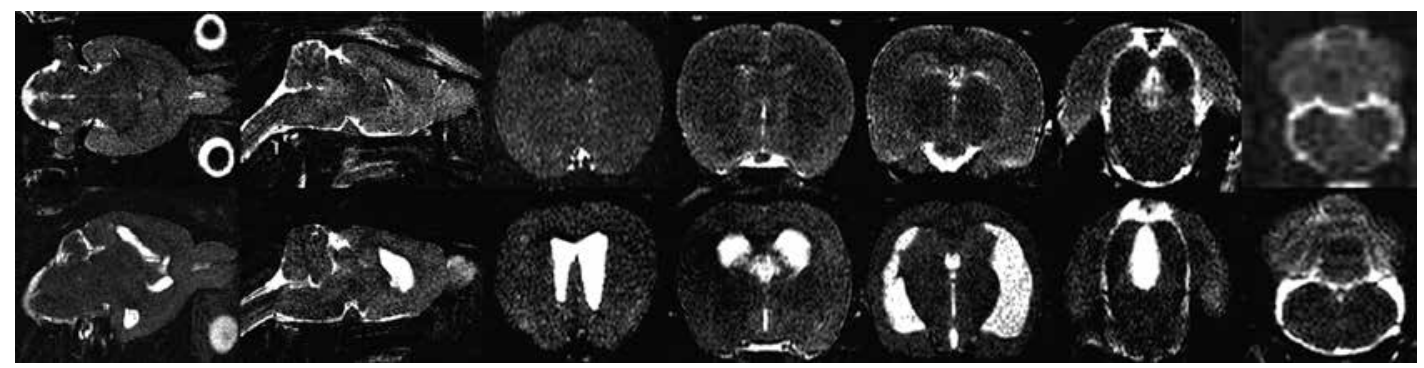

B
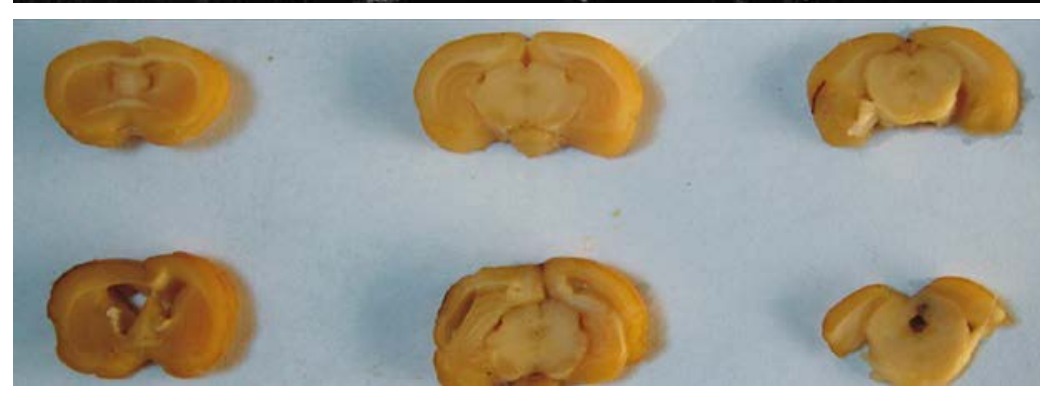

Hydrocephalus
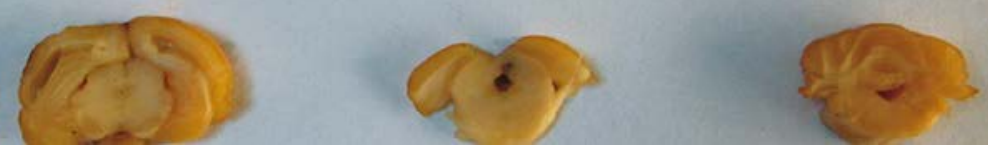

Fig. 2. A) Representative magnetic resonance imaging (T2-weighted) of rat brains in the horizontal plane (first column), in the mid-sagittal plane (second column) and at the coronal level of the lateral ventricle frontal horn (third column), optic chiasm (fourth column), lateral ventricle temporal horn (fifth column), cerebral aqueduct (sixth column) and basal cistern (seventh column). Row 1 shows control rats; the ventricles, cerebral aqueduct and cisterna magna are barely visible. Row 2 shows hydrocephalic rats at the $16^{\text {th }}$ day after kaolin injection; the ventricles, cerebral aqueduct and cisterna magna show significant enlargement. B) General specimens of rat cerebral tissue with consecutive coronal sections. Row 1 - control group specimens showing no expansion of the ventricular system. Row 2 - experimental group represents significantly expanded lateral ventricle anterior horns (first column) and temporal horns (second column), midbrain aqueduct (third column) and fourth ventricle (fourth column), and compression, thinning, fracture and distortion of the corpus callosum and cortical tissue. 
proliferation of hypertrophic astrocytes and disorder of organization structure; the ventricular wall was completely deprived of ependymal cells, which were lying loosely in the ventricular lumen (Fig. 4).

\section{Discussion}

The basic research on hydrocephalus has not experienced a breakthrough since the early $20^{\text {th }}$ century. One of the main causes may be that we have not established a uniform and particularly effective animal model. Most studies have remained at the stage of animal model establishment. Regarding hydrocephalic animal model establishment, there are too many methods. This method of kaolin injection into the lateral ventricle to induce communicating hydrocephalus has a lot of advantages theoretically, but has not yet been widely applied. Therefore, we tried to construct and optimize the model of hydrocephalus after intraventricular kaolin injection for use in adult rats and provide a description of behavioral and histological changes.

Excluding unexpected death in the process of anesthesia or surgical trauma, all rats with kaolin injection into the lateral ventricle developed mod-

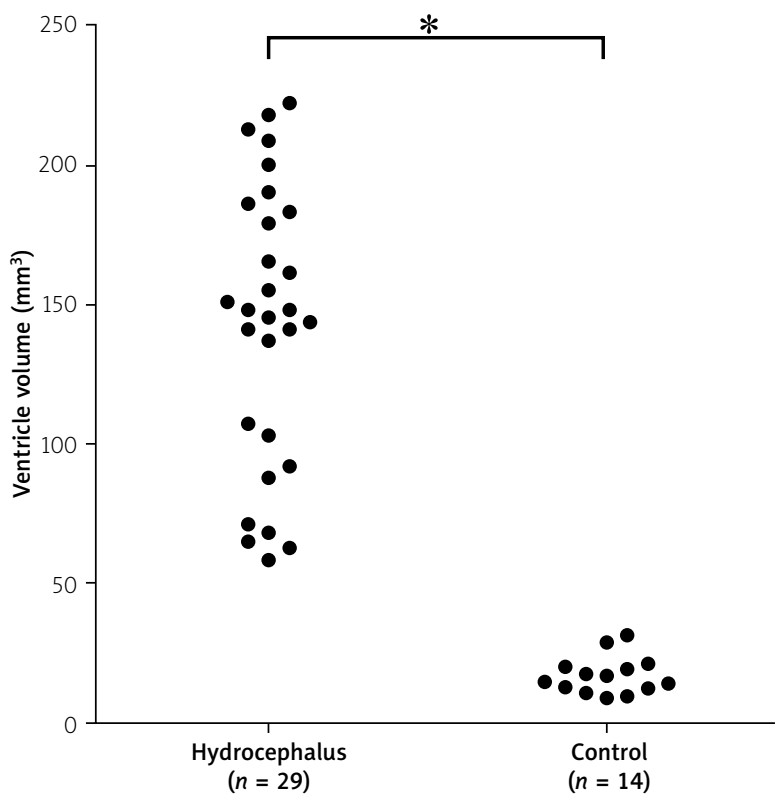

Fig. 3. Ventricular volumes were measured by NIH Image. Hydrocephalic rats exhibited significant ventricular dilation compared to controls (hydrocephalus - median value: $147.95 \mathrm{~mm}^{3}$, range: 58.51-222.27 $\mathrm{mm}^{3}$; controls - median value: $15.65 \mathrm{~mm}^{3}$, range: $\left.8.78-31.52 \mathrm{~mm}^{3}\right)$. The asterisk indicates $p<0.05$ (Mann-Whitney $U$ test).

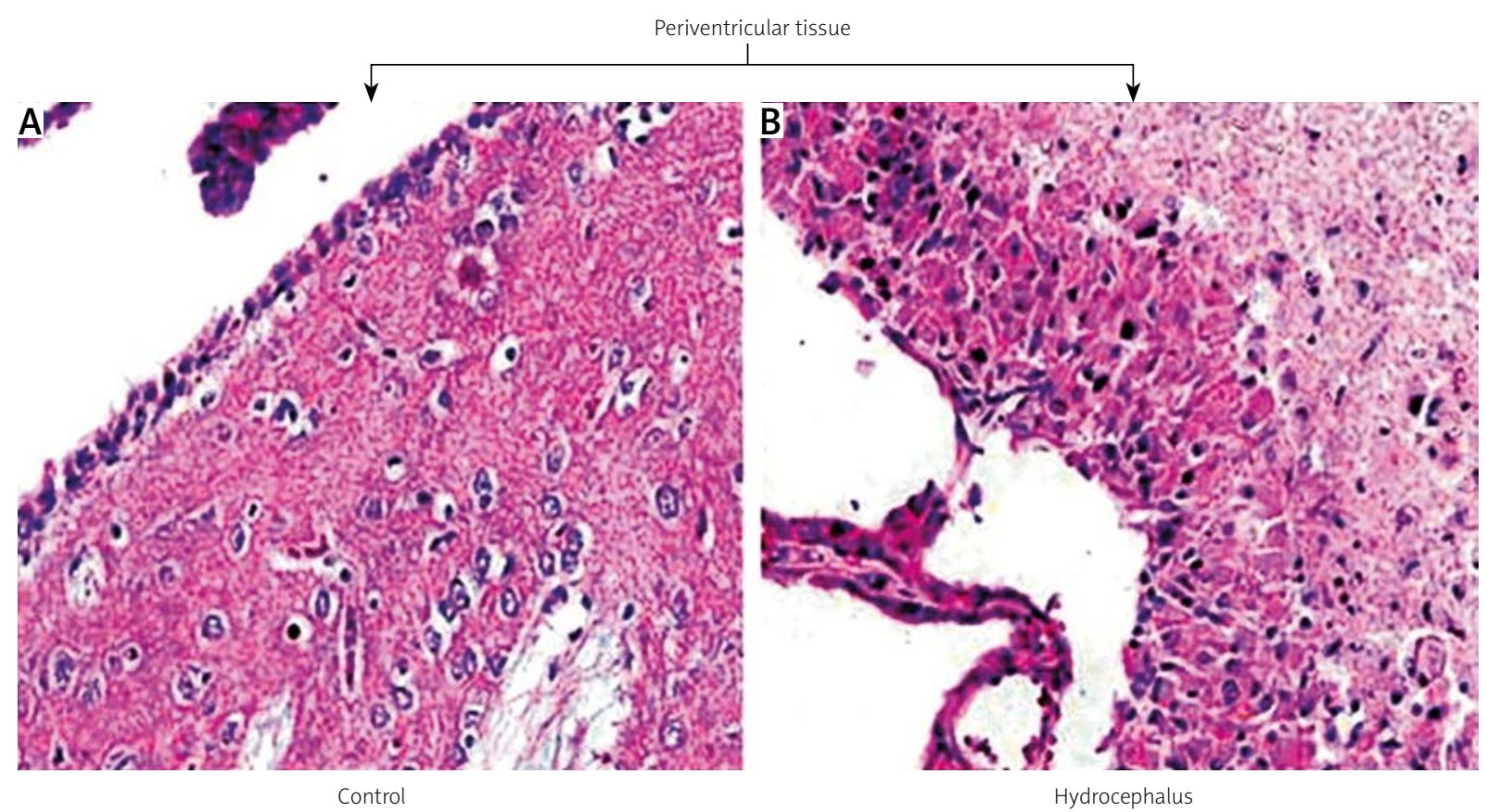

Fig. 4. Representative images of H\&E staining of the lateral ventricle. Compared with the controls, the hydrocephalic animals showed advanced periventricular reactive astrogliosis with proliferation of hypertrophic astrocytes and disorder of organization structure; the ventricular wall was completely deprived of ependymal cells, which were lying loosely in the ventricular lumen (magnification, ×400). 
erate to severe hydrocephalus according to MRI assay, and they could maintain long-term survival. However, using the technique of kaolin injection into the cistern magna of rodents, the acute mortality of animals was up to 10-50\% [21,26,29]; and Bloch et al. reported that they had an about $20 \%$ acute mortality rate and $80 \%$ of the survivors died by 10 days [2]. The lower concentration kaolin suspensions after ultrasonic emulsification for about 15 minutes are less viscous, spread more easily through the CSF pathways and easily form a communicating hydrocephalus. Compared to kaolin injection into the cistern magna, the low concentration kaolin suspension injection into the lateral ventricle does not cause comprehensive adhesion of the cistern magna, facilitating CSF sample collection from the cistern magna, and does not lead to brain stem injury or a sharp rise of intracranial pressure, so the acute mortality rate is low. If we want to obtain more severely hydrocephalic animals and reduce animal death simultaneously, the method can be used again one week after injection. In addition, hydrocephalic animals exhibited a significant lag in weight gain, and severely hydrocephalic rats gradually lost weight. This phenomenon is in agreement with a previous report [21].

Magnetic resonance imaging and general specimens of rat cerebral tissue revealed that animals with severe hydrocephalus exhibited obvious expansion of ventricles, and thinning, distortion and compression of the cortical tissue and the corpus callosum. Hematoxylin and eosin (H\&E) staining revealed that the hydrocephalic samples had high levels of glial cell hyperplasia, cell edema and liquefaction and organization structure disorder in the vicinity of the lateral ventricle. These changes in the hydrocephalus may directly cause deleterious effects and clinical symptoms such as dizziness, headache, gait disturbance, urinary incontinence and cognitive deficits/dementia that seriously threaten the patient's quality of life and the prognosis $[5,7,9,20]$. Impairments of gait and balance are the most common and the earliest symptoms of hydrocephalus. Patients may initially complain about dizziness, difficulty in walking on a slope or stairs, and difficulty in getting up from or sitting down on a chair; in the late stage of hydrocephalus, motor deficits are very common. Urinary incontinence in hydrocephalus results from detrusor hyperactivity owing to the partial or total absence of central inhibitory control. Patients initially suffer from increased urinary frequency; later developments are urge incontinence and, finally, permanent urinary incontinence. The cognitive deficits of hydrocephalus are mainly due to sub-cortical frontal dysfunction. The main clinical manifestations of cognitive deficits/dementia are as follows: psychomotor slowing, impaired attention and concentration, slowing and reduced precision of fine motor performance, changes of mood and personality, short-term memory impairment, indifference and bradyphrenia, etc. About one-third of children with hydrocephalus have been reported to have an IQ of $<70$ and another third to have an IQ in the low normal range of 70-85 [19]. It has also been shown that children with congenital hydrocephalus have difficulties in learning and memory, problems that are not explained by low IQ scores alone. The decline of these above-mentioned functions is deemed "fronto-subcortical dementia" due to dysfunction of the frontal lobe and subcortical structures [3,31], and was partly observed in the course of our experiment, suggesting that the frontal cortex is severely affected in hydrocephalus [17]. In this study, disturbed spatial learning and memory were observed using the MWM test, which is consistent with the findings of previous studies [28].

The concentrations of kaolin reported in the literature were 25\%, 20\%, 10\%, etc., and 25\% kaolin was widely applied $[11,18,21,29]$. To determine the experimental concentration of kaolin, several different concentrations of kaolin (1\%, 3\%, 5\%, 10\%, $20 \%, 25 \%, 40 \%$ and $50 \%$ ) were used in a small-sample experimental design in the preliminary experiment. The results showed that the high concentration kaolin suspensions were difficult to dissolve in saline, and tended to deposit more readily in the lateral ventricle in general specimens and spread with more difficultly through the CSF pathways, which may create a physical obstruction; moreover, they had a relatively low success rate and high acute mortality. However, the lower concentration kaolin suspensions, which can be dissolved and emulsified in saline by ultrasonic dissolution and did not jam the microsyringe, were less viscous and spread more easily through the CSF pathways, and had high success rates, low mortality rates and fewer complications. It seemed that the concentrations of $3 \%$ and $5 \%$ kaolin showed no significant difference in success rate. So we chose the $3 \%$ kaolin. In addition, we also attempted to use injection of autologous blood 
from the caudal artery into the lateral ventricle to construct a model of hydrocephalus, but the success rate was very low.

Based on our experience, we conclude that this experimental hydrocephalus model, in which kaolin is injected into the lateral ventricle of adult rats, is viable because of its many advantages (including simplicity, cheapness, high success rate, low mortality, success in developing a communicating hydrocephalus and controllable hydrocephalic severity, etc.) and may be widely used. At the same time, we also provide a description of behavioral and histological changes, etc.

\section{Conclusions}

In summary, we have provided detailed information on the use of kaolin injection into the lateral ventricle to induce hydrocephalus in adult rats, and this model is similar to the clinical disease. In the next stage, we will explore further the biochemical changes in the cerebral tissue and CSF.

\section{Acknowledgments} versity.

This study was partially supported by Xiamen Uni-

\section{Disclosure}

The authors report no conflict of interest.

\section{References}

1. Aquilina K, Hobbs C, Cherian S, Tucker A, Porter H, Whitelaw A, Thoresen M. A neonatal piglet model of intraventricular hemorrhage and posthemorrhagic ventricular dilation. J Neurosurg 2007; 107: 126-136.

2. Bloch O, Auguste KI, Manley GT, Verkman AS. Accelerated progression of kaolin-induced hydrocephalus in aquaporin-4-deficient mice. J Cereb Blood Flow Metab 2006; 26: 1527-1537.

3. Bonelli RM, Cummings JL. Frontal-subcortical circuitry and behavior. Dialogues Clin Neurosci 2007; 9: 141-151.

4. Chen X, Huang X, Li B, Zhao Z, Jiang L, Huang C, Lu Y. Changes in neural dendrites and synapses in rat somatosensory cortex following neonatal post-hemorrhagic hydrocephalus. Brain Res Bull 2010; 83: 44-48.

5. Dalen K, Bruaroy S, Wentzel-Larsen T, Laegreid LM. Intelligence in children with hydrocephalus, aged 4-15 years: a population-based, controlled study. Neuropediatrics 2008; 39: 146150.

6. Davis LE. Communicating hydrocephalus in newborn hamsters and cats following vaccinia virus infection. J Neurosurg 1981; 54: 767-772.
7. Del Bigio MR. Neuropathological changes caused by hydrocephalus. Acta Neuropathol 1993; 85: 573-585.

8. Del Bigio MR. Pathophysiologic consequences of hydrocephalus. Neurosurg Clin N Am 2001; 12: 639-649.

9. Del Bigio MR. Cellular damage and prevention in childhood hydrocephalus. Brain Pathol 2004; 14: 317-324.

10. Del Bigio MR. Neuropathology and structural changes in hydrocephalus. Dev Disabil Res Rev 2010; 16: 16-22.

11. Deren KE, Packer M, Forsyth J, Milash B, Abdullah OM, Hsu EW, McAllister JP, 2nd. Reactive astrocytosis, microgliosis and inflammation in rats with neonatal hydrocephalus. Exp Neurol 2010; 226: 110-119.

12. Factora R, Luciano M. Normal pressure hydrocephalus: diagnosis and new approaches to treatment. Clin Geriatr Med 2006; 22: 645-657.

13. Fernell E, Hagberg B, Hagberg G, von Wendt L. Epidemiology of infantile hydrocephalus in Sweden. III. Origin in preterm infants. Acta Paediatr Scand 1987; 76: 418-423.

14. Fiori MG, Sharer LR, Lowndes HE. Communicating hydrocephalus in rodents treated with beta,beta'-iminodipropionitrile (IDPN). Acta Neuropathol 1985; 65: 209-216.

15. Hayashi N, Leifer DW, Cohen AR. Chronologic changes of cerebral ventricular size in a transgenic model of hydrocephalus. Pediatr Neurosurg 2000; 33: 182-187.

16. Hwang YS, Shim I, Chang JW. Anxiety responses and neurochemical changes in a kaolin-induced rat model of hydrocephalus. J Neurosurg Pediatr 2011; 7: 401-407.

17. Kiefer M, Unterberg A. The differential diagnosis and treatment of normal-pressure hydrocephalus. Dtsch Arztebl Int 2012; 109: 15-25; quiz 26.

18. Li J, McAllister JP, 2nd, Shen Y, Wagshul ME, Miller JM, Egnor MR, Johnston MG, Haacke EM, Walker ML. Communicating hydrocephalus in adult rats with kaolin obstruction of the basal cisterns or the cortical subarachnoid space. Exp Neurol 2008; 211: 351-361.

19. Lindquist B, Carlsson G, Persson EK, Uvebrant P. Learning disabilities in a population-based group of children with hydrocephalus. Acta Paediatr 2005; 94: 878-883.

20. Lindquist B, Persson EK, Uvebrant P, Carlsson G. Learning, memory and executive functions in children with hydrocephalus. Acta Paediatr 2008; 97: 596-601.

21. Lopes Lda S, Slobodian I, Del Bigio MR. Characterization of juvenile and young adult mice following induction of hydrocephalus with kaolin. Exp Neurol 2009; 219: 187-196.

22. McAllister JP, 2nd, Chovan P. Neonatal hydrocephalus. Mechanisms and consequences. Neurosurg Clin N Am 1998; 9: 73-93.

23. Nagra G, Wagshul ME, Rashid S, Li J, McAllister JP, 2nd, Johnston M. Elevated CSF outflow resistance associated with impaired lymphatic CSF absorption in a rat model of kaolin-induced communicating hydrocephalus. Cerebrospinal Fluid Res 2010; 7: 4.

24. Ohmiya M, Fukumitsu H, Nitta A, Nomoto H, Furukawa Y, Furukawa S. Administration of FGF-2 to embryonic mouse brain induces hydrocephalic brain morphology and aberrant differentiation of neurons in the postnatal cerebral cortex. J Neurosci Res 2001; 65: 228-235. 
25. Owler BK, Pena A, Momjian S, Czosnyka Z, Czosnyka M, Harris NG, Smielewski P, Fryer T, Donvan T, Carpenter A, Pickard JD. Changes in cerebral blood flow during cerebrospinal fluid pressure manipulation in patients with normal pressure hydrocephalus: a methodological study. J Cereb Blood Flow Metab 2004; 24: 579-587.

26. Park YS, Park SW, Suk JS, Nam TK. Development of an acute obstructive hydrocephalus model in rats using N-butyl cyanoacrylate. Childs Nerv Syst 2011; 27: 903-910.

27. Shihab N, Davies E, Kenny PJ, Loderstedt S, Volk HA. Treatment of hydrocephalus with ventriculoperitoneal shunting in twelve dogs. Vet Surg 2011; 40: 477-484.

28. Shim I, Ha Y, Chung JY, Lee HJ, Yang KH, Chang JW. Association of learning and memory impairments with changes in the septohippocampal cholinergic system in rats with kaolin-induced hydrocephalus. Neurosurgery 2003; 53: 416-425.

29. Slobodian I, Krassioukov-Enns D, Del Bigio MR. Protein and synthetic polymer injection for induction of obstructive hydrocephalus in rats. Cerebrospinal Fluid Res 2007; 4: 9.

30. Tada T, Zhan H, Tanaka Y, Hongo K, Matsumoto K, Nakamura T. Intraventricular administration of hepatocyte growth factor treats mouse communicating hydrocephalus induced by transforming growth factor beta1. Neurobiol Dis 2006; 21: 576-586.

31. Tekin S, Cummings JL. Frontal-subcortical neuronal circuits and clinical neuropsychiatry: an update. J Psychosom Res 2002; 53 : 647-654.

32. Wiesmann M, Koedel U, Bruckmann H, Pfister HW. Experimental bacterial meningitis in rats: demonstration of hydrocephalus and meningeal enhancement by magnetic resonance imaging. Neurol Res 2002; 24: 307-310.

33. Williams MA, McAllister JP, Walker ML, Kranz DA, Bergsneider M, Del Bigio MR, Fleming L, Frim DM, Gwinn K, Kestle JR, Luciano MG, Madsen JR, Oster-Granite ML, Spinella G. Priorities for hydrocephalus research: report from a National Institutes of Health-sponsored workshop. J Neurosurg 2007; 107: 345-357.

34. Zhao K, Sun H, Shan Y, Mao BY, Zhang H. Cerebrospinal fluid absorption disorder of arachnoid villi in a canine model of hydrocephalus. Neurol India 2010; 58: 371-376. 